\title{
Quasi-Optical Design Of A Millimeter Wave Imaging System
}

\author{
Faeze jadidi ${ }^{1}$, Abdollah Eslamimajd ${ }^{* 2}$, Alireza Erfaniyan ${ }^{3}$, Seyed \\ Hossein Mohseni Armaki ${ }^{3}$
}

1: Malek Ashtar University of Technology, Faculty of Electrical \& Computer, Department of Electrical Engineering, Lavizan, Tehran, Iran, 158751774, jadidi@mut.ac.ir

2: Malek Ashtar University of Technology, Faculty of Electrical \& Computer, Department of Electrical Engineering, Lavizan, Tehran, Iran, 158751774, a_eslamimajd@mut-es.ac.ir

3: Malek Ashtar University of Technology, Faculty of Electrical \& Computer, Department of Electrical Engineering, Lavizan, Tehran, Iran, 158751774

\begin{abstract}
In this paper, we report complete design and simulation of a quasi-optical millimeter wave imaging system with Fresnel lens and horn Antenna using ZEMAX and FEKO softwares, respectively. It is much easier to make a Fresnel lens at millimeter wavelengths compared to spherical and aspherical lenses. The use of Fresnel lens to focus millimeter wave radiation greatly reduces the thickness and also the weight of the focusing element from $25 \mathrm{Kg}$ to $4.5 \mathrm{Kg}$. A horn antenna with Gaussian profile and corrugated walls at the central frequency of $94 \mathrm{GHz}$ for feeding this system is designed. The symmetry of the designed corrugated Gaussian horn radiation pattern in $\mathrm{E}$ and $\mathrm{H}$ orthogonal planes, its wide bandwidth as well as its low side lobe levels make it a good candidate for feeding a $\mathrm{W}$ band millimeter-wave imaging system. The designed quasioptical imaging system has low weight with high resolution and can be used to detect hidden objects within a distance of 5 meters with $30 \mathrm{~mm}$ resolution in $\mathrm{W}$ band at $94 \mathrm{GHz}$ central frequency.
\end{abstract}

Key Words:

Fresnel lens, millimeter wave imaging, aspheric lens, corrugated Gaussian horn antenna.

\section{1- Introduction}

Millimeter wave imaging systems have many applications. This technology is capable of imaging under adverse climatic conditions and enables the detection of hidden objects such as polymers [1]. New threats, including plastic and ceramic rifles and knives are not detectable by metal detectors, which can only detect metal targets. The effectiveness of these detectors varies depending on the amount, direction and type of metals. In addition, metal detectors cannot distinguish between items such as glasses, belts, keys, and real threats, leading to false alarms [2]. Due to the unique features of millimeter waves in the 
electromagnetic spectrum including high penetration and low energy, millimeter wave technology is expanding rapidly. This technology is safe for living beings compared to Xrays and has the potential to penetrate strongly into plastics, cardboard, textiles and other materials used for packing and are reflected by metallic materials. This feature is desired for security and detection of hidden weapons, luggage of passengers at airports and border points $[3,4]$.

Millimeter waves cover a wavelength range of 1-10 $\mathrm{mm}$, which corresponds to a frequency range of 30-300 GHz [5]. Regarding the atmospheric transition, there are two optimal frequency bands, Q (35 GHz) and W $(94 \mathrm{GHz})$ bands, for use in millimeter wave imaging. Frequency selection is based on a simple rule: select the lower frequency for greater penetration depth and optics with larger aperture, and/or the higher frequency for better resolution. Compared to $\mathrm{Q}$ band, $\mathrm{W}$ band has a better image quality and spatial resolution controlled by the diffraction limit. The distance of the millimeter wave imaging system from the target is usually several meters [6].

The history of millimeter wave technology dates back to 1890, but the first important activity in this field was conducted in 1930 [7]. Ditchfield and England (1955) introduced the first millimeter wave imaging system in the UK [8]. Since then, the technology has continued with rapid advances in recent years [9]. The dielectric lens or reflector can be used for imaging systems [10]. Of course, each of the two methods of refraction and reflection has advantages and disadvantages. The reflective systems are light in weight, but due to their small number of components and interconnection, they have less field of view than equivalent refraction systems, and the installation of mirrors is even more difficult. Refraction systems are direct and more efficient due to no central blockage [11]. Also, dielectric lens manufacturing unlike reflectors, requires less precise tolerance and uses inexpensive plastic materials [12]. So they are good options to be used as focusing elements in the millimeter wave band. The lens material should have a low loss and mass density and medium dielectric constant $\left(\varepsilon_{-} \mathrm{r}=2-4\right)$ [13]. Millimeter wave band lenses are usually made of materials like high density polyethylene (HDPE), silicon, polystyrene, rexolite and/or teflon. HDPE is a cheap material and can be easily machined using the computer cut $[14,15]$. The uniform dielectric lens with two surfaces is equivalent to two reflectors, because each surface is equivalent to a degree of freedom in design. Forming both surfaces allows designers to design a lens that corrects aberrations [16].

The performance of highly accurate optical systems using spherical optics is limited by aberrations. Using aspherical optics, geometric aberrations can be reduced or removed. New methods of manufacturing allow producing high-precision aspherical surfaces [17]. Since quasi-optical imaging systems should reduce the blurring effect in order to have an acceptable sharpness, an aspherical lens can be used [6].

A 43-cm diameter HDPE aspherical transition system at $89 \mathrm{GHz}$ was capable of imaging the object at a distance of $3.5 \mathrm{~m}$ with a resolution of $28 \mathrm{~mm}$ developed by Zhou et al. (2015) and Chen et al. reported a similar system with $35 \mathrm{~mm}$ resolution at a distance of $3.5 \mathrm{~m}[1,18]$. The development of HDPE transition system with a diameter of $50 \mathrm{~cm}$ at 94 $\mathrm{GHz}$ using one-dimensional array of receivers has also been reported in 2011[19]. A focal array imaging system using an acrylic lens with a diameter of $20 \mathrm{~cm}$ and resolution of $2 \mathrm{~cm}$ at a distance of $1 \mathrm{~m}$ at 75-95 GHz frequency has been also made in 2011[20].

In the millimeter wave band where we have to use large lenses because of the limit of diffraction, the system weight is too high [6]. Each time when thinner, lighter, and easier 
to manufacture systems required to focal the incoming radiation, Fresnel lenses are preferred to conventional refraction lenses. Fresnel lenses use diffraction as a method of collecting electromagnetic waves at the focal point. In this type of lens, the stepped discrete pattern first proposed by Lord Rayleigh, can achieve phase correction. According to this theory, different methods have been proposed and the desired phase correction has been achieved [21-23,15].

A $600 \mathrm{GHz}$ Fresnel lens has been designed for active and passive imaging by Chen et al. (2007) [24]. Design and manufacturing of zone plate Fresnel lenses with opaque and transparent area at millimeter wavelengths have been reported by Leon et al. (2014) [25]. Also, the use of dielectric Fresnel lens for imaging has been investigated at a frequency of 75 to $110 \mathrm{GHz}$ in 2017[26].

In quasi-optical systems, the free space energies focused by the lens to a horn antenna which transmits it to the detector. There are different types of horn antennas that their advantages and disadvantages should be taken into account when designing a quasioptical system [27]. In high-performance applications in the millimeter wave area, such as security imaging, radar, and radio astronomy, corrugated horn antennas are commonly used for feeding due to their high ray symmetry, relatively low side lobe levels, and low cross-polarization [28].

The process of quasi-optical system design involves the design of primary optics (selection of focal element) and secondary optics (selection of horn profile and wall surface features) according to the system requirements. In this study, the primary optics is designed using an aspherical lens that has a better spot diagram and corrected aberrations than the spherical lens. Then, due to the high weight of the aspherical dielectric lens, a grooved Fresnel lens has been simulated by software ZEMAX to replace it. A corrugated Gaussian profile horn has been also designed at a central frequency $94 \mathrm{GHz}$ as secondary optics and simulated using electromagnetic software FEKO. This horn can be used with a lens (spherical, aspherical or Fresnel lens) with f-number of 1.2, to meet the requirements of the system. The system is capable of imaging an object with a spatial resolution of 30 $\mathrm{mm}$ at a distance of $5 \mathrm{~m}$.

\section{2- Result and Discussion}

\section{2-1 Aspherical lens}

The spatial resolution in imaging applications is finally limited to diffraction. The angle is limited to the diffraction corresponding to the central zone of the diffraction pattern, which comprises $84 \%$ of radiation distribution. The optical system resolution according to Rayleigh's criterion in Equation (1) equals:

$s=R \frac{1.22 \lambda}{D}$

Where $\lambda$ is the central frequency of operation, $\mathrm{R}$ is the imaging distance, $\mathrm{D}$ is the diameter of the input aperture and $\mathrm{s}$ is the resolution required at this distance for imaging [11]. According to Rayleigh's criterion for achieving a resolution of $30 \mathrm{~mm}$ at a distance of $5 \mathrm{~m}$ in $94 \mathrm{GHz}$, the aperture diameter size should be about $65 \mathrm{~cm}$.

The designed optical system is a transmission dielectric aspherical lens, simulated by software ZEMAX and illustrated in Fig. 1. HDPE lens dielectric material with refractive 
index of 1.5147 at $94 \mathrm{GHz}$ is suitable for imaging (at $94 \mathrm{GHz}, \tan \delta=0.0003$ and $\varepsilon=2.2$ ). HDPE is not listed in ZEMAX тм Glass catalog list and was entered in the frequency range of $45-145 \mathrm{GHz}$ using "appropriate coefficient data" and Conrady's formula according to Equation (2):

$n(\lambda)=n_{0}+\frac{A}{\lambda}+\frac{B}{\lambda^{3.5}}$

Conrady's formula is a well-known equation for changing refractive index of a material in the wavelength area that uses three pairs of wavelength-refractive index data to create continuous capability [25].

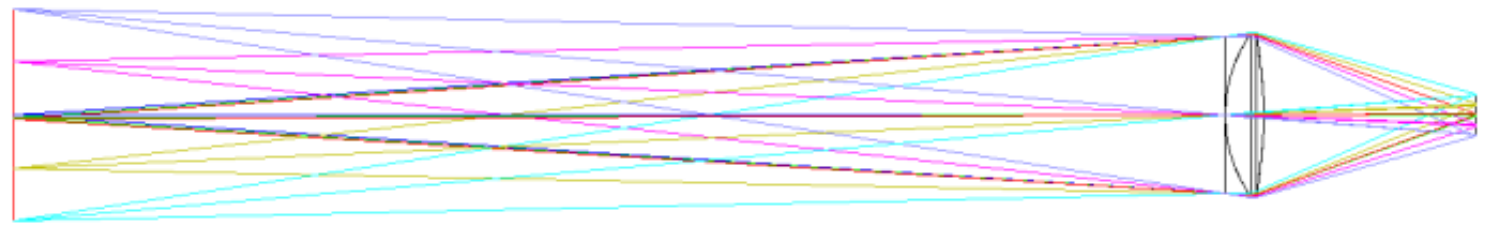

Fig. 1: Overview of the designed aspherical lens.

The formula of the aspherical lens surface according to Equation (3) is:

$Z=\frac{c^{-1} r^{2}}{1+\sqrt{1-(1+k) c^{-2} r^{2}}}+\alpha_{1} r^{2}+\alpha_{2} r^{4}+\alpha_{3} r^{6}+\alpha_{4} r^{8}+\alpha_{5} r^{10}$

Where $\mathrm{c}$ is curvature, $\mathrm{r}$ is the radial coordinate, and $\mathrm{k}$ is the conic constant.1Table 1 shows the optimized values of aspherical surface constants of simulated lens according to ZEMAX. D and d, the diameter and thickness of the lens are 650 and 71 millimeter respectively. According to entered parameters like mass density in ZEMAX, this lens weighs $25 \mathrm{~kg}$.

Table 1: Aspherical lens design constants.

\begin{tabular}{|c|c|c|}
\hline Parameter & Surface 1 & Surface 2 \\
\hline $\mathrm{C}$ & 921.542 & -915.071 \\
\hline $\mathrm{K}$ & 1.791 & -71.404 \\
\hline$\alpha_{1}$ & $2.38 \mathrm{e}-4$ & $-1.343 \mathrm{e}-6$ \\
\hline$\alpha_{2}$ & $1.746 \mathrm{e}-9$ & $-1.139 \mathrm{e}-10$ \\
\hline$\alpha_{3}$ & $-8.464 \mathrm{e}-15$ & $-6.672 \mathrm{e}-16$ \\
\hline$\alpha_{4}$ & $-4.151 \mathrm{e}-21$ & $-9.193 \mathrm{e}-23$ \\
\hline
\end{tabular}

Figure 2 shows the spot diagram of the designed system for 6 different positions in the field of view covering an area of $50 \times 50 \mathrm{~cm}^{2}$. In this system, the aberrations have been corrected with optimization program in ZEMAX and the root mean square size of the spot at the end of the field of view is approximately $3 \mathrm{~mm}$. The image quality curves of spot diagram, focused energy, and MTF as well as their analysis show that the designed system has a high image quality. 


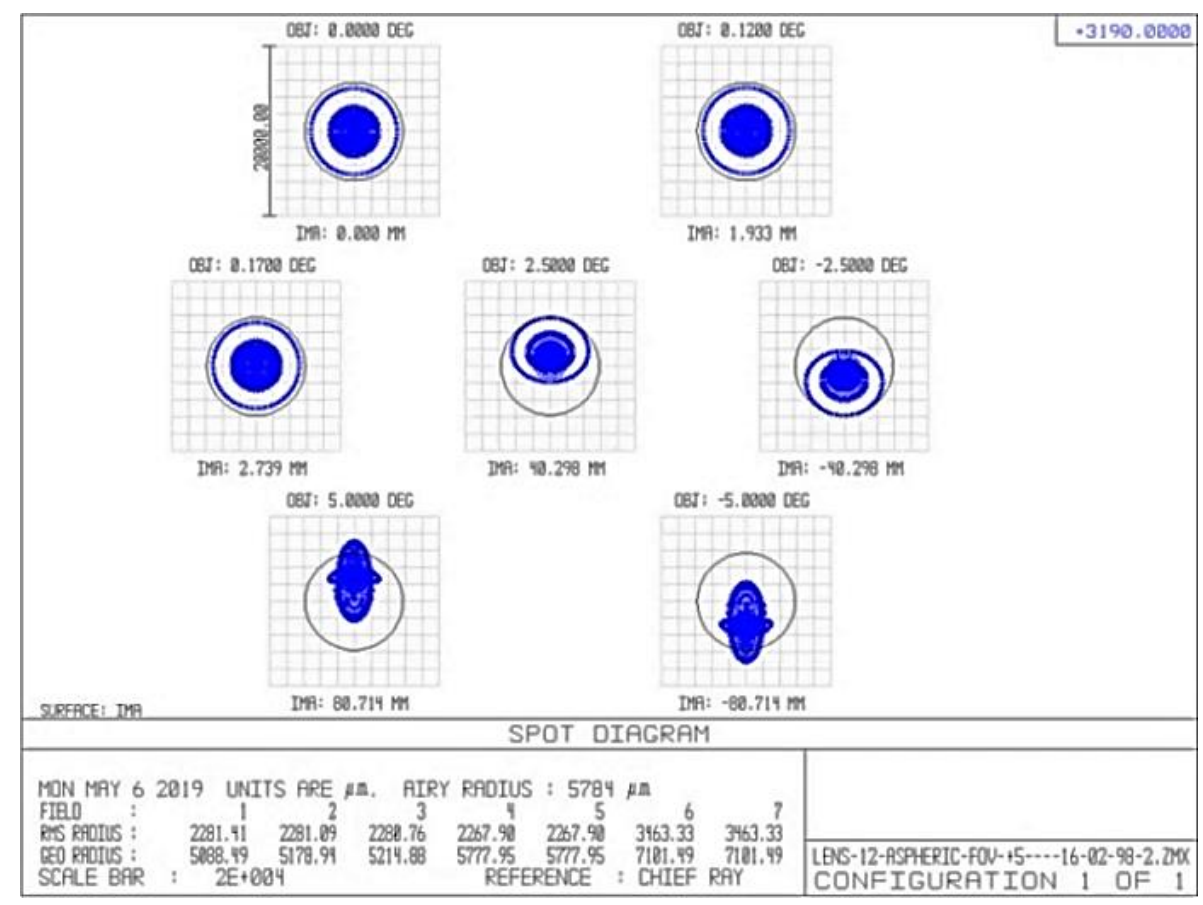

Fig. 2: Spot diagram of the designed aspherical lens.

\section{2-2- Fresnel lens}

A zone plate is a tool for image formation whose mechanism is not refraction, but rather diffraction in the aperture rings. The interference of the diffracted radiation generates the image. The spherical wave-front can be modified by using different material permittivities (Fig. 3.(a)) or phase correction areas (Fig. 3.(b)) [22,29].

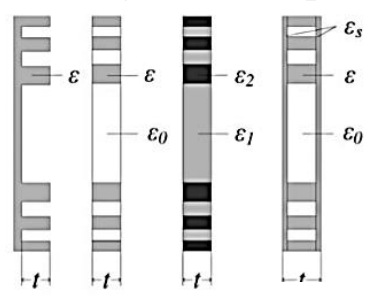

(a)
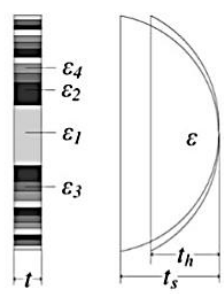

(20)
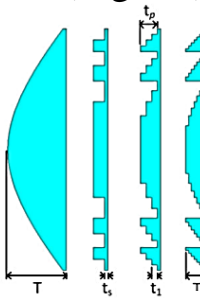

Fig. 3: Example of a Fresnel lens: (a) Multi-dielectric Fresnel zone plate lens and (b) grooved Fresnel zone plate lens [22,29].

When the wave-front passes through the lens, the lens imposes a phase difference on the wave. The result is nearly a spherical wave-front that converges on the focal point of the lens [30]. By applying simple rules during the design process, the Fresnel lens antenna can achieve high efficiency with low side lobe level. In order to keep the complexity acceptable, the phase transfer steps can be used when the phase change reaches as half phase, quarter phase and $\mathrm{P}$ phase. So, each time the phase change reaches 180,90 , and $2 \pi / \mathrm{p}$, Fresnel lens compensates the phase. The $\mathrm{p}$ parameter corresponds to the number of compensations made during a 360 degree phase change. Since this compensation is not complete, the lens will have limited efficiency [31-33]. In a Fresnel lens, each groove forms a prism, so Fresnel lens is made up of a set of prisms. The grooves near the center 
of Fresnel lens are almost flat and shallow, and the grooves near the outside points have deep and sharp angles [34-36].

All diffracted lens areas are involved in focusing the light at the right point. For the design of the diffraction lens, the transition points should be calculated for each area, which is a correct multiple of the wavelength relative to the focal point of the lens (Fig. 4).

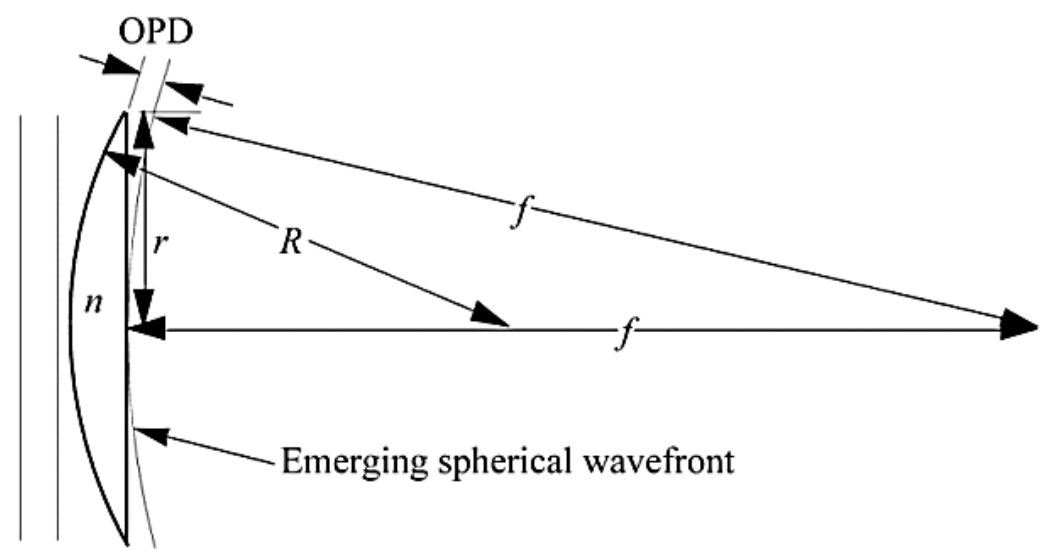

Fig. 4: Geometry used to determine the locations of transition points for a diffraction lens[30].

In lens of focal length $\mathrm{f}$, which operates at wavelength $\lambda(2 \pi$ phase, the change equals one $\lambda$ optical path difference), the radius of the area $p$ using Pythagorean Theorem, where the phase change equals $2 \pi$ is equal to:

$r_{p}^{2}=2 f p \lambda$

The phase difference caused by the lens is the difference between the input wave at any point and the phase delay due to the passing through the lens at distance $r$ from the axis equals:

$\Delta \varphi(r)=2 \pi(n-1) r^{2} / 2 R \lambda$

The optical path difference at different points of the wave-front equals to:

$O P D=\Delta \varphi(r) \lambda / 2 \pi$

Software ZEMAX calculates the location of the transition points for symmetric lens phase profile (even power of radius) by minimizing the aberrations. The phase function in this program is as follows:

$\varphi(r)=2 \pi / \lambda O P D=2 \pi / \lambda\left(A_{1} r^{2}+A_{2} r^{4}+A_{3} r^{6}+A_{4} r^{8}\right)$

Fresnel lens substrate designed by ZEMAX is a flat disk. Its profile is made of radial flat surfaces whose endpoints are defined by SAG expression [30].

The given two-dimensional Fresnel lens design is illustrated in Fig. 5. Its input aperture has a radius of $330 \mathrm{~mm}$. The number of grooves in this Fresnel lens is 16 . The central thickness of the designed Fresnel lens is $12.7 \mathrm{~mm}$. Also, the spherical surface radius of this lens is $778.9 \mathrm{~mm}$ and its conical coefficient is equal to -1.115 . 


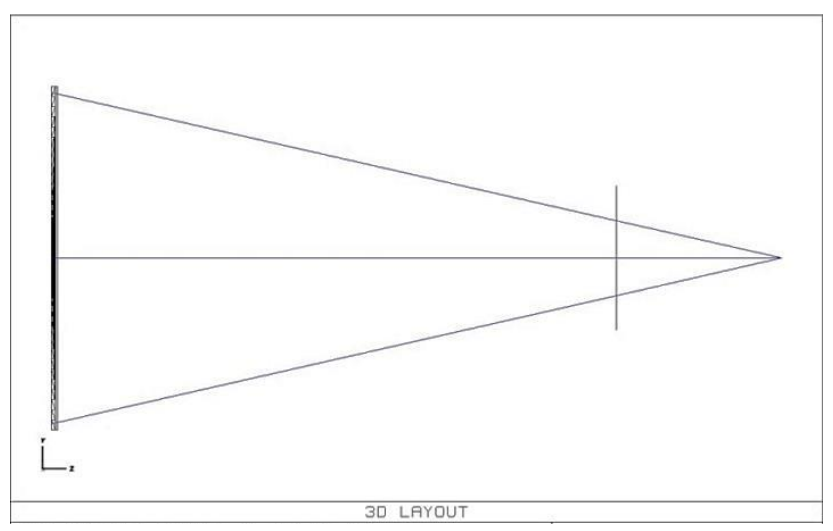

Fig. 5: Designed Fresnel lens.

For the design of this type of lens in ZEMAX, the non-sequential component of Fresnel was used and its 3D design is shown in Fig. 6. This lens weighs $4.5 \mathrm{~kg}$, which is sharply reduced compared to the corresponding spherical and aspherical lens and can be used in a low-weight millimeter wave imaging system with a resolution of $30 \mathrm{~mm}$ at a distance of $5 \mathrm{~m}$.

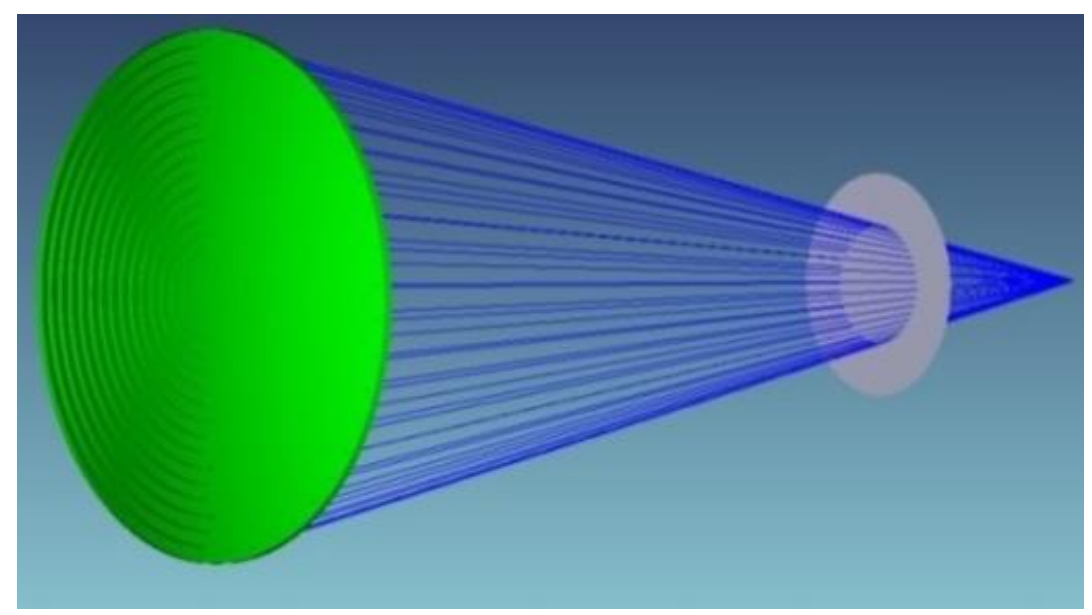

Fig. 6: 3D Design of Fresnel lens.

Figure 7 shows the optimized Fresnel lens spot diagram. The RMS of the lens spot's radius is $5.626 \mathrm{~mm}$. In order to optimize this lens, the image surface is set as the global coordinate reference, and the default merit function has been used with RMS of the spot's radius of all configurations as convergence criteria. 


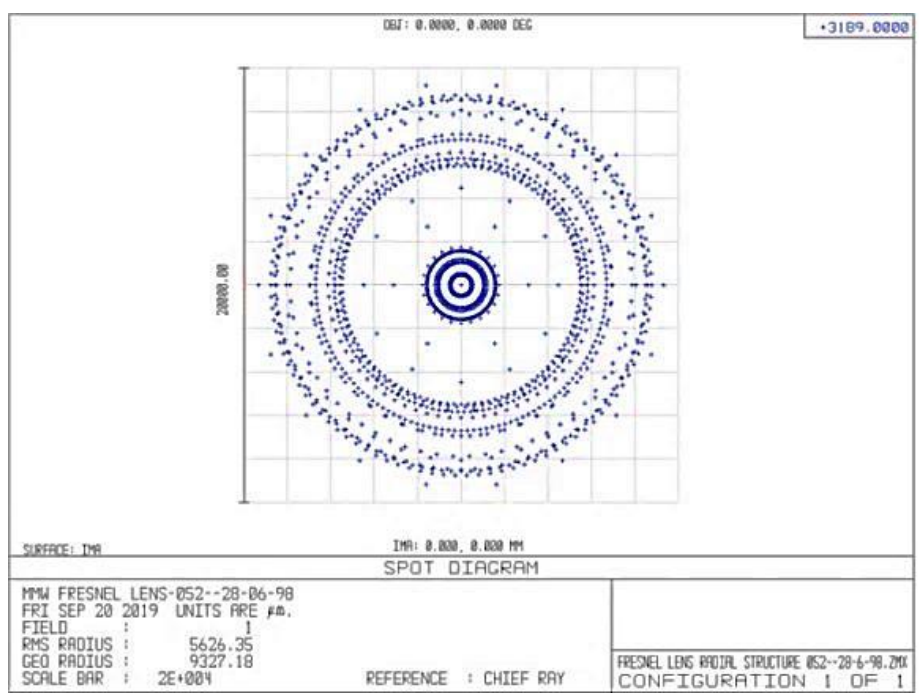

Fig. 7: Optimized Fresnel lens spot diagram.

\section{2-3- Design and simulation of secondary optics}

For the design of secondary optics, one should specify horn profile and its wall type (flat or corrugated). It is so important to match the feed horn beam width to the system Fnumber (F\#) to optimize the performance of the system. The usual values of the optimum feed taper (a measure of the ratio of the power reduction at the opening edge to the power on the axis) are in the range of -10 to $-13 \mathrm{~dB}$ (Fig. 8). If $\theta_{-10 \mathrm{~dB}} \gg \theta_{0}$ spillover loss will occur and some power will be lost. If $\theta_{-10 \mathrm{~dB}} \ll \theta_{0}$ the amplitude taper loss and phase error loss result in deviation from amplitude and constant phase in the opening field [37].

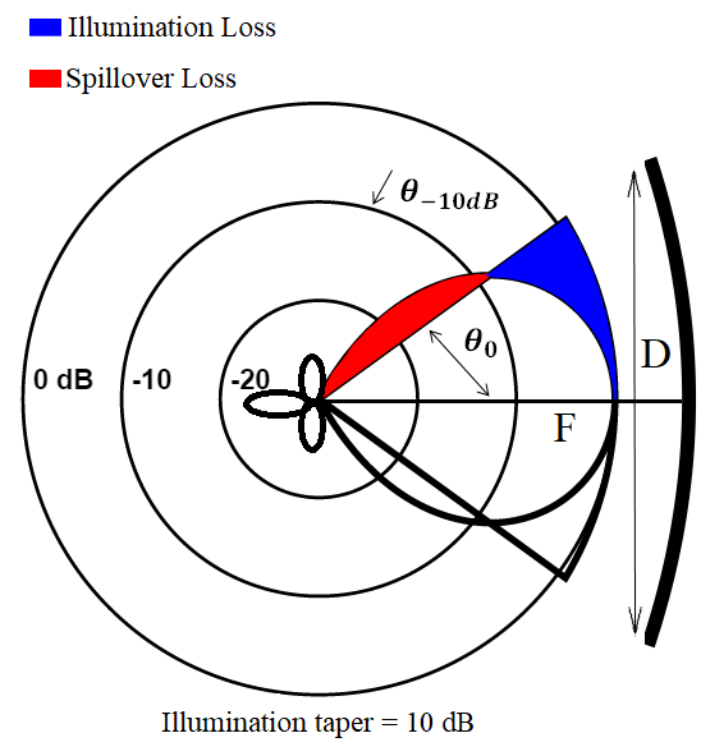

Fig. 8: Spillover loss of combined lens and Horn antenna. 
The performance of horn antenna with the linear opening wall is improved by profiling the wall [37]. Horn profile can be sine, tangential, exponential, hyperbolic, polynomial, etc [38]. Horn antenna of Gaussian profile provides a smooth transition from waveguide to opening, which improves the matching between antenna and free space. This better matching ensures better radiation pattern and more bandwidth and increases total efficiency of the system [39]. Using horn antennas with Gaussian profile, various parameters such as beam diffraction, bandwidth, side lobe levels, transverse polarization, orientation, gain, efficiency, and etc. can be optimized. In Gaussian horn antennas, the main features of the cone horn antenna radiation pattern are preserved, and the side lobe levels as well as the cross-polarization surface are significantly reduced [39,40].

Next step after selecting the wall profile of the horn is choosing features of the wall (flat or corrugated). The flat-wall horn antennas have problems that are solved by wall corrugation. These problems include uneven beam widths and uneven phase centers in two orthogonal plates, more side lobes on E-plate than H-plate, and diffraction from Eplate walls that will cause the back lobe [16].

First we designed an optimized Gaussian profile horn with flat wall in FEKO software for feeding the desired optical system. The structure of this horn is shown in Fig. 9.

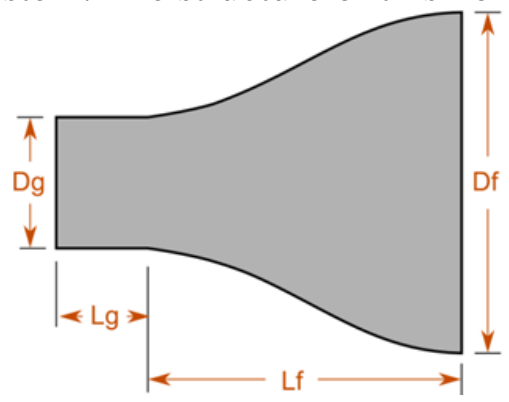

Fig. 9: Schematic of the designed Gaussian profile horn with flat wall.

The optimized parameters for this horn are listed in Table 2 where Dg is the waveguide diameter, $\mathrm{Lg}$ is the waveguide length, Df is Gaussian profile final diameter and Lf is Gaussian profile length.

Table 2: Aspherical lens design constants.

\begin{tabular}{|c|c|c|c|}
\hline Dg & Lg & Df & Lf \\
\hline 16.43 & 19.14 & 3.18 & 2.55 \\
\hline
\end{tabular}

Figure 10 shows the radiation pattern of the flat wall Gaussian horn at the $94 \mathrm{Ghz}$ frequency in two orthogonal planes. 


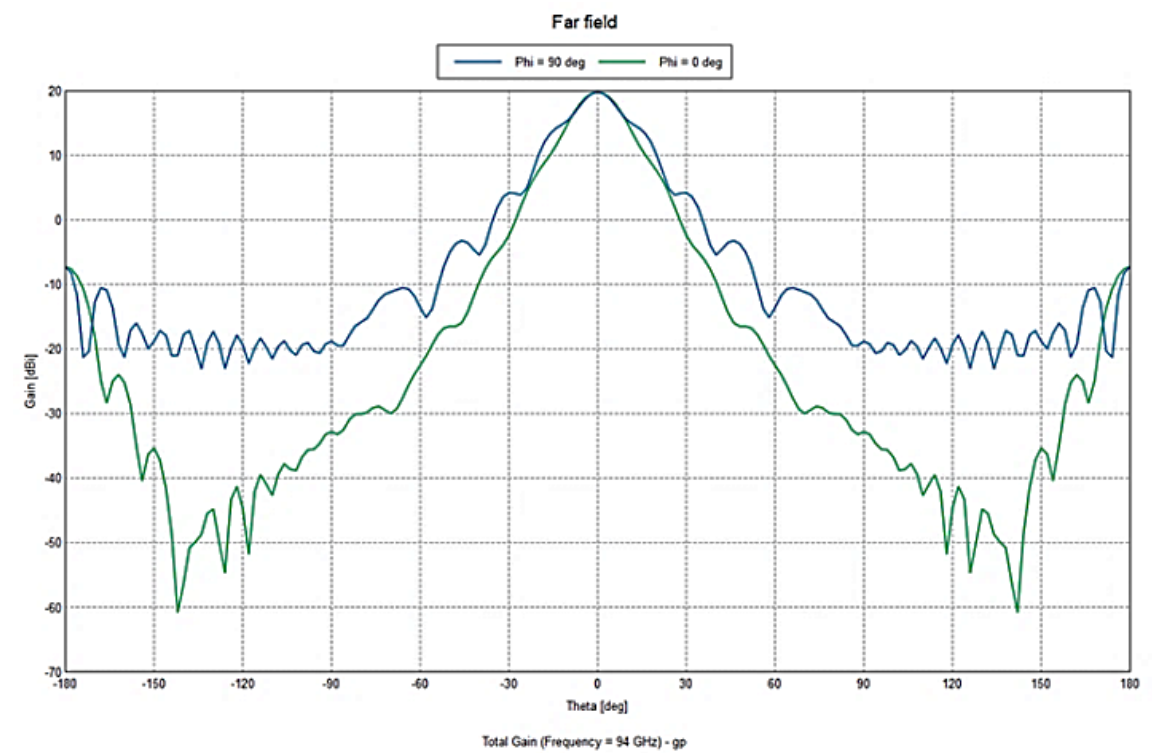

Fig. 10: Radiation pattern of flat wall Gaussian profile horn at frequency of $94 \mathrm{GHz}$.

Then we designed Gaussian profile horn with a corrugated wall with reduced side lobes. Side lobes would cause to receive radiation from unwanted directions. Also symmetrical pattern means that the aberrations are reduced and more transition of focused energy with low reflection from the aperture can reach the detector. The horn is well designed to match the primary optics.

\section{2-4- Design a corrugated Gaussian feeding horn}

The structure of this horn is designed according to the instructions given in reference [41] and the obtained parameters optimized in FEKO software. This horn, as shown in Fig. 11, comprises a circular waveguide with transition from a flat linear section to a corrugated linear section and is used as a mode converter. The depth of the grooves in the cone convertor section starts with the initial value approximately half the wavelength at the maximum frequency and ends at one quarter the wavelength at the central frequency.

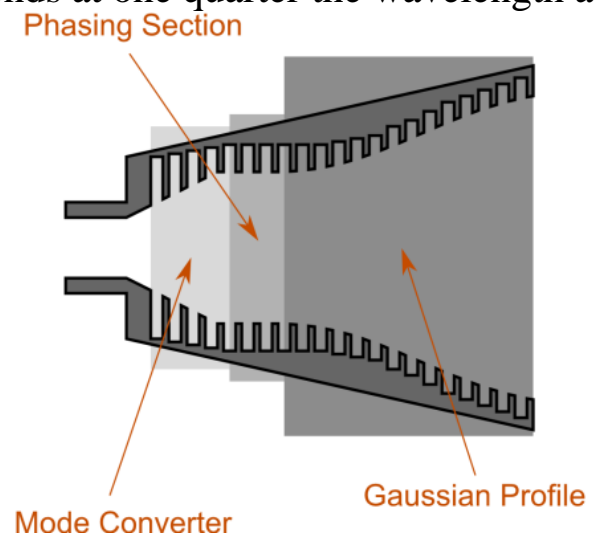

Fig. 11: Schematic of the corrugated Gaussian horn. 
The mode converter is a phasing section that acts as the feed of Gaussian profile section. The depth of all slots after the converter is equal. Each of these sections are discussed below.

\section{2-4-1 Mode converter}

Gaussian horn antenna should be fed by a purely $\mathrm{HE}_{11}$ mode. Therefore, in the horn neck area, the impedance converter is used to accommodate flat single-mode waveguide $\mathrm{TE}_{11}$ with corrugated waveguide [42]. This mode converter usually starts with a single-mode circular flat propagator waveguide, $\mathrm{TE}_{11}$ and terminates at the opening diameter required for phasing section used to feed horn antenna with Gaussian profile. The proposed parameters for the corrugated cone horn (Fig. 12) are listed in Table 3.

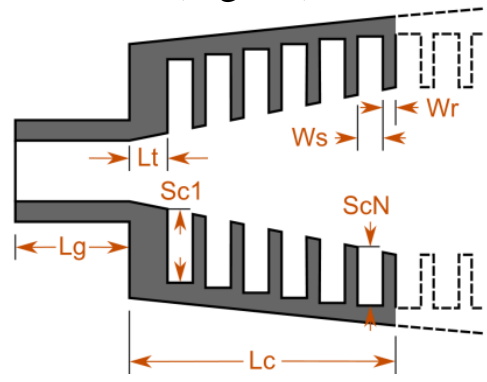

Fig. 12: Mode converter design.

In Table 3, 2a is the waveguide diameter, $\mathrm{Lg}$ is the waveguide length, $\mathrm{Lt}$ is transition area length, Sc1 is the depth of the first groove in the converter, $\mathrm{ScN}$ is the depth of the last groove in the converter, Ws is the groove width, $\mathrm{Wr}$ is the edge width, Lc is the axial length of the converter section and $\mathrm{Nc}$ is the number of grooves in the converter section.

Table 3: Mode converter parameters ( $\mathrm{mm})$.

\begin{tabular}{|c|c|c|c|c|c|c|c|c|}
\hline $2 \mathrm{a}$ & $\mathrm{Lg}$ & $\mathrm{Lt}$ & $\mathrm{Sc} 1$ & $\mathrm{ScN}$ & $\mathrm{Ws}$ & $\mathrm{Wr}$ & $\mathrm{Lc}$ & $\mathrm{Nc}$ \\
\hline 2.55 & 3.18 & 0.797 & 1.27 & 0.79 & 0.53 & 0.13 & 8.23 & 10 \\
\hline
\end{tabular}

\section{2-4-2 Phasing section}

Due to the compression of the corrugated horn antenna in the first section, the orientation is low and the phase centers on $\mathrm{E}$ and $\mathrm{H}$ plates do not match. Therefore, a phasing section is used to overlap the phase centers in the desired frequency band. This section also improves the combination of $\mathrm{TE}_{11}$ and $\mathrm{TM}_{11}$ modes. Phasing section is shown in Fig. 13 and the design parameters for this section are given in Table 4.

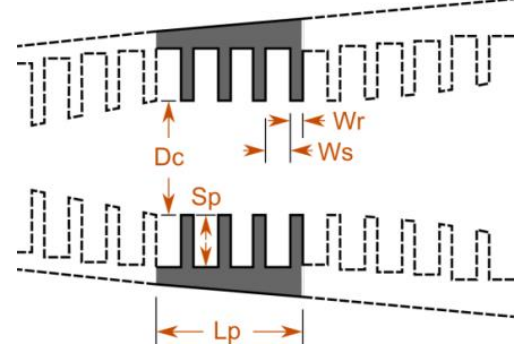

Fig. 13: Phasing section design. 
In Table 4, Dc is phasing section diameter, $\mathrm{Sp}$ is the groove depth in phasing section, $\mathrm{Lp}$ is the length of phasing section, $\mathrm{Np}$ is the number of grooves in phasing section, and Ws and $\mathrm{Wr}$ are the groove and edge width, respectively.

Table 4: Phasing section parameters (mm).

\begin{tabular}{|c|c|c|c|}
\hline Dc & Sp & Lp & $\mathrm{Np}$ \\
\hline 9.17 & 0.79 & 2.65 & 4 \\
\hline
\end{tabular}

\section{2-4-3 Gaussian section parameters}

The design parameters of this section according to Fig. 14 are given in Table 5.

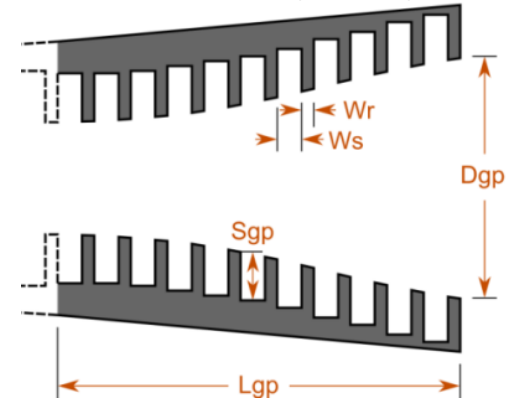

Fig. 14: Corrugated Gaussian section design.

In Table 5, Dgp is Gaussian profile final diameter, Sgp is depth of Gaussian profile, Lgp is Gaussian profile length, Ngp is the number of grooves of Gaussian profile section and $\mathrm{Ws}$ and $\mathrm{Wr}$ are the groove and edge width, respectively.

Table 5: Gaussian section parameters ( $\mathrm{mm}$ ).

\begin{tabular}{|c|c|c|c|}
\hline Dgp & Sgp & Lgp & Ngp \\
\hline 13.9 & 0.79 & 9.96 & 15 \\
\hline
\end{tabular}

3.1.4 The results of Gaussian corrugated horn simulation

The most important parameter for our design is the symmetry of radiation pattern and low side lobe levels. Figure 15 shows 3D radiation pattern of the designed horn. Its gain is about $20 \mathrm{dBi}$. It has a very symmetrical pattern in both orthogonal planes and the side lobes reduced so much relative to other common horns in this wavelength range. 


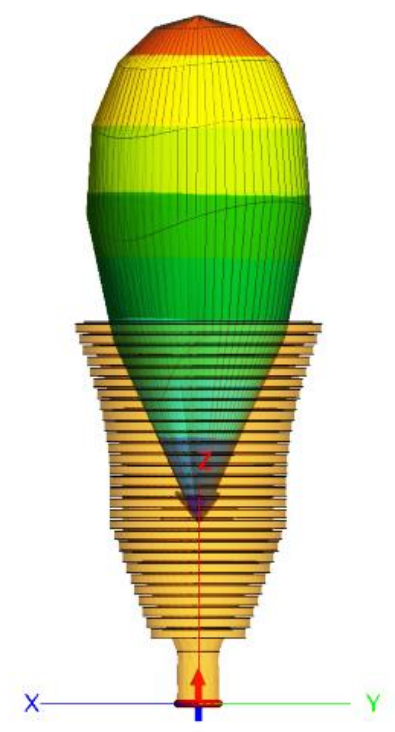

Fig. 15: Radiation pattern of the designed corrugated Gaussian horn at frequency of 94 $\mathrm{GHz}$.

Figure 16 shows the radiation pattern of the corrugated horn at the desired design frequency in $\mathrm{E}$ and $\mathrm{H}$ planes. At the optimal frequency band, the levels of side lobes are below $-38.5 \mathrm{~dB}$, which is a very good result.

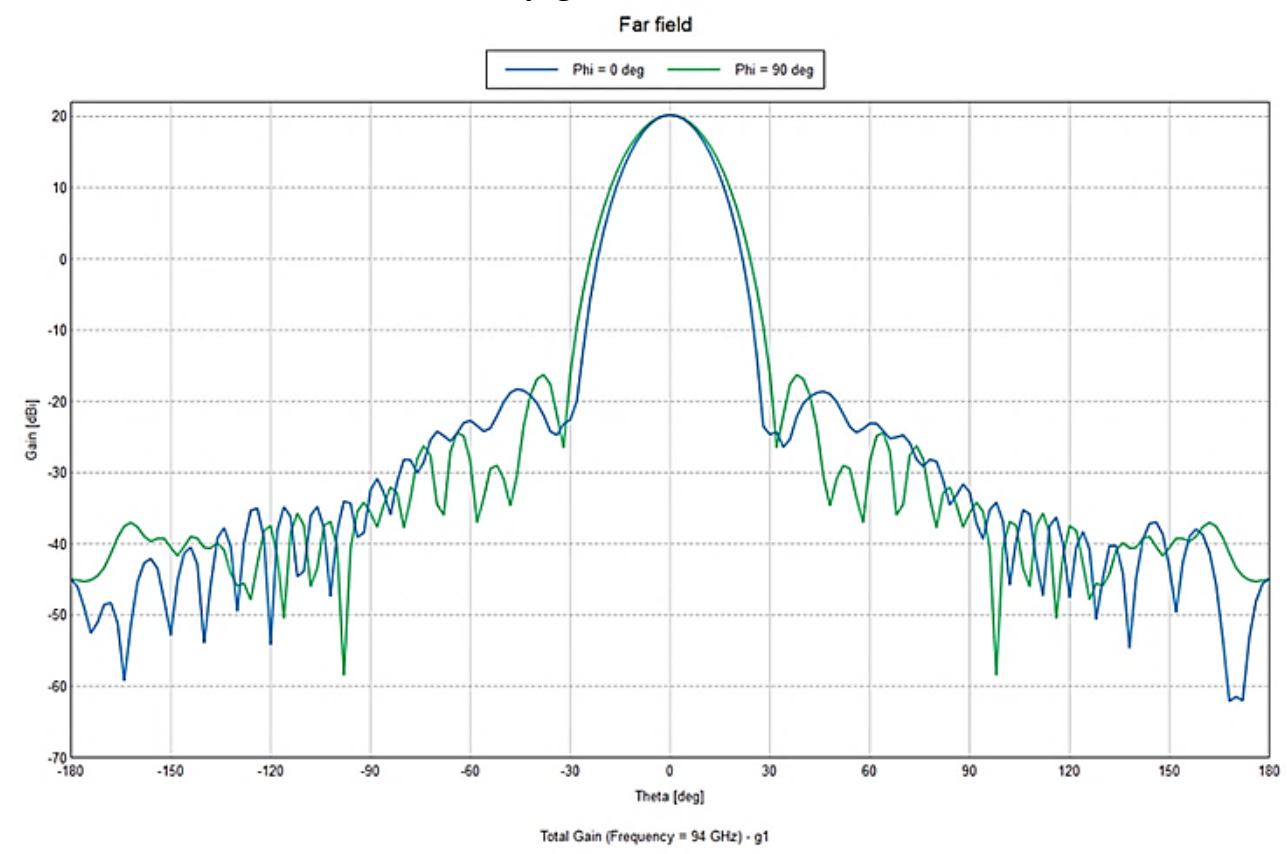

Fig. 16: Radiation pattern of the far field at a frequency of $94 \mathrm{GHz}$.

The pattern symmetry is very good and the horn $-10 \mathrm{~dB}$ beamwidth is about 40 degrees, making it appropriate for use in the designed quasi-optical transition system.

\section{3- Conclusion}


In this study, we have designed and simulated a millimeter wave imaging system including a lens and a feedhorn. The lens aperture diameter is $65 \mathrm{~cm}$, capable of imaging an object with a resolution of $30 \mathrm{~mm}$ in a 5-meter imaging range in central frequency of $94 \mathrm{GHz}$. The aspherical lens designed for the millimeter wave camera is a high quality system with well-corrected aberrations. The image quality curves also show the high quality of the designed system. In addition, due to the ease of manufacturing Fresnel lens in this wavelength band and in order to reduce the weight and volume of the imaging system, a Fresnel lens has been designed and simulated. The optimized corrugated Gaussian horn has been designed with a gain of about $20 \mathrm{~dB}$ with a 10-dB beam width of $40^{\circ}$ on $\mathrm{E}$ and $\mathrm{H}$ planes aimed to provide the proper axial pattern symmetry. According to the results of calculations and simulations, it can be concluded that a combination of Fresnel lens and Gaussian corrugated horn is very suitable and this complete quasioptical system can be used for millimeter wave imaging with high resolution to identify hidden objects in security imaging applications.

\section{Availability of data and materials:}

'Not applicable'.

\section{Competing interests:}

'The authors declare that they have no competing interests'.

\section{Funding:}

'Not applicable'.

\section{Authors' contributions:}

Faeze Jadidi carried out the initial studies, designed of optical system and horn and drafted the manuscript. Abdollah Eslamimajd participated in the design of the study and performed the statistical analysis. Alireza Erfaniyan and Seyed Hossein Mohseni Armaki conceived of the study, and participated in its design and helped to draft the manuscript. All authors read and approved the final manuscript.

\section{Acknowledgements:}

'Not applicable'.

\section{Endnotes:}

'Not applicable'.

\section{Reference}


1. Zhou, J, Chen, Q, Zhang, Y, Fan, Y, Da Xu, K: Aspheric dielectric lens antenna for millimeter-wave imaging system. In:Asia-Pacific Microwave Conference (APMC), 3, 1-3, IEEE, Nanjing, China (2015). doi:10.1109/APMC.2015.7413384.

2. Sheen, DM, McMakin, DL, Hall, TE: Three-dimensional millimeter-wave imaging for concealed weapon detection, IEEE Trans. Microw. Theory Tech. 49, 1581-1592 (2001). doi:10.1109/22.942570

3. Zhang, C, Deng, C: Passive terahertz imager. In 2011 International Conference on Infrared, Millimeter, and Terahertz Waves, IEEE, Houston, TX, USA (2011). doi: 10.1109/irmmw-THz.2011.6105113

4. Clark, SE, Lovberg, JA, Martin, CA, Kolinko, VG: Passive millimeter-wave imaging for airborne and security applications, Proc. SPIE 5077, Passive Millimeter-Wave Imaging Technology VI and Radar Sensor Technology VII, (2003). https://doi.org/10.1117/12.484871

5. Sheen, DM, McMakin, DL, Hall, TE: Near field imaging at microwave and millimeter wave frequencies. In: 2007 IEEE/MTT-S International Microwave Symposium, 1693-1696, IEEE, Honolulu, HI, USA (2007). doi: 10.1109/MWSYM.2007.380033

6. Kim, WG, Moon, NW, Singh, MK, Kim, HK, Kim, YH: Characteristic analysis of aspheric quasi-optical lens antenna in millimeter-wave radiometer imaging system, Appl. Opt. 52, 1122-1131 (2013). doi: 10.1364/AO.52.001122

7. Wiltse, JC: History of millimeter and submillimeter waves, IEEE Trans. Microw. Theory Tech. 32, 1118-1127 (1984). doi:10.1109/TMTT.1984.1132823

8. Giles, DA, Linfield Edmund, H., Pepper, M, Appleby, R:Passive millimetre-wave imaging and how it differs from terahertz imaging, Phil. Trans. R. Soc. A.362379-393 (2004). https://doi.org/10.1098/rsta.2003.1323

9. Appleby, R, Anderton, RN: Millimeter-wave and submillimeter-wave imaging for security and surveillance, Proceedings of the IEEE, 95(8), 1683-1690 (2007). doi: 10.1109/JPROC.2007.898832.

10. Lettington, A, Alexander, N, Dunn, D: A new opto-mechanical scanner for millimeter and sub-millimeter wave imaging, in Passive Millimeter-Wave Imaging Technology VIII, 5789, 16-24, SPIE, Orlando, Florida, United States (2005). https://doi.org/10.1117/12.597745.

11. Fischer, RE, Tadic-Galeb, B, Yoder, PR, Galeb, R, Kress, BC, McClain, SC, et al., Optical system design, 2 edition, McGraw-Hill Education (2008).

12. Minin, IV, Minin, OV: Basic principles of Fresnel antenna arrays, 19, Springer Science \& Business Media (2008).

13. O'Sullivan, CM, Murphy, JA: Field guide to terahertz sources, detectors, and optics, SPIE (2012). https://doi.org/10.1117/3.952851

14. Jinghui, Q, Zhong, Z, Kai, L, Gaofei, L, Fei, X: Design and measurement of quasioptics for millimeter wave imaging system, 2009 IEEE International Workshop on Imaging Systems and Techniques, Shenzhen, 2009, 132-135, doi:10.1109/IST.2009.5071618. 
15. Walsby, E, Wang, S, Xu, J, Yuan, T, Blaikie, R, Durbin, S: Multilevel silicon diffractive optics for terahertz waves, J. Vac. Sci. Technol B. 20, 2780-2783, (2002). doi:10.1116/1.1518021

16. Milligan, TA: Modern antenna design, 2 edition, Wiley-IEEE Press (2005).

17. Henselmans, R: Non-contact measurement machine for freeform optics, Technische Universiteit Eindhoven, Citeseer (2009).

18. Chen, Q, Fan, Y, Zhou, J, Song, K: Design of Quasi-Optical Lens Antenna for WBand Short Range Passive Millimeter-Wave Imaging, J. Comput. Commun. 3, p. 93, (2015). doi: 10.4236/jcc.2015.33016.

19. Yeom, S, Lee, DS, Son, JY, Jung, MK, Jang, Y, Jung, SW: Real-time outdoor concealed-object detection with passive millimeter wave imaging, Opt. Express. 19, 2530-2536 (2011). doi: 10.1364/OE.19.002530.

20. Li, CM, Huang, CY, Chang, LY, Yu, YC, Nien, CC, Tarng, JH: Development of a compact total power passive millimeter-wave imaging system, in 2011 IEEE International Symposium on Radio-Frequency Integration Technology, 153-156, Beijing, China (2011). doi: 10.1109/RFIT.2011.6141793

21. Keshavarz, A, Soltanzadeh, MJ: Designing the optimal Fresnel lenses by using Zemax software, J. optoelectron. nanostructures. 3, 87-96 (2018).

22. Rodríguez, J, Hristov, HD, Grote, W: Fresnel zone plate and ordinary lens antennas: Comparative study at microwave and terahertz frequencies, In: 2011 41st European Microwave Conference, 894-897, IEEE, Manchester, UK (2011).

23. Garrett, JE, Wiltse, JC: Fresnel zone plate antennas at millimeter wavelengths, Int. J. Infrared Mill. 12, 195-220 (1991).

24. Chen, C, Prather, DW, Siegel, PH: Design of a $600 \mathrm{GHz}$ fresnel lens antenna for passive and active imaging, In: 2007 IEEE Antennas and Propagation Society International Symposium, 4385-4388, Honolulu, HI, USA (2007).

25. León Fernández, GL, Herrán Ontañón, F, Munoz, M, Las Heras Andrés, FL, Hao, Y: Millimeter-wave offset Fresnel zone plate lenses characterization, Prog. Electromagn. Res. C. 54, 125-131 (2014).

26. Jouadé, A: Millimeter-wave radar imaging systems: focusing antennas, passive compressive devicefor MIMO configurations and high resolution signal processing, Ph.D. Thesis, University of Rennes (2017).

27. Murphy, JA, Trappe, N, Withington, S: Gaussian beam mode analysis of partial reflections in simple quasi-optical systems fed by horn antennas. Infrared physics \& technology 44(4), 289-297 (2003). https://doi.org/10.1016/S13504495(03)00127-0

28. Gundersen, J, Wollack, E: Millimeter wave corrugated platelet feeds. In: Journal of Physics: Conf. Ser 2009.

29. Hristov, HD, Rodriguez, JM: Design equation for multidielectric Fresnel zone plate lens. IEEE Microwave and Wireless Components Letters 22(11), 574-576 (2012). doi: 10.1109/LMWC.2012.2224099

30. O'Shea, DC, Suleski, TJ, Kathman, AD, Prather, DW:Diffractive optics: design, fabrication, and test, 62, SPIE press (2004) https://doi.org/10.1117/3.527861.

31. Black, DN, Wiltse, JC: Millimeter-wave characteristics of phasecorrecting Fresnel zone plates. IEEE Transactions on Microwave Theory and Techniques, 35(12):1122-1129, (1987). doi:10.1109/TMTT.1987.1133826 
32. Hristov, HD, HAJ Herben, M: Millimeter-wave Fresnel-zone plate lens and antenna. IEEE Transactions on Microwave Theory and Techniques, 43(12):2779-2785, (1995).

33. Jouade, A, Bor, J, Himdi, M, Lafond, O: Millimeter-wave fresnel zone plate lens with new technological process. International Journal of Microwave and Wireless Technologies, 1-6, 008 (2016). doi: https://doi.org/10.1017/S1759078716000854

34. Miller, O, McLeod, J, Sherwood, W: Thin sheet plastic Fresnel lenses of high aperture, J. Opt. Soc. Am. 41(11), 807-815 (1951). https://doi.org/10.1364/JOSA.41.000807

35. Davis, A, Kühnlenz, F:Optical design using Fresnel lenses: Basic principles and some practical examples, Opt. Photon. 2, 52-55 (2007).

36. Erismann, F:Design of a plastic aspheric Fresnel lens with a spherical shape, Opt. Eng. 36, 988-992 (1997).

37. Volakis, JL: Antenna engineering handbook (4th ed.). New York: McGraw-Hill (2007).

38. Granet, C:Profile options for feed horn design, in 2000 Asia-Pacific Microwave Conference. Proceedings (Cat. No. 00TH8522), 1448-145, IEEE, Sydney, NSW, Australia (2000).

39. Kishk, A, Lim, CS:Comparative Analysis Between Conical and Gaussian Profiled Horn Antennas-Abstract, J. ELECTROMAGNET. WAVE. 17, 599-600 (2003).

40. De Miguel-Hernández, J, Hoyland, RJ: Fundamentals of horn antennas with low cross-polarization levels for radioastronomy and satellite communications. Journal of Instrumentation 14(08), R08001 (2019). doi:10.1088/1748-0221/14/08/R08001 41. Granet, C, James, GL:Design of corrugated horns: A primer, in IEEE Antennas and Propagation Magazine, vol. 47, no. 2, 76-84, (2005). doi: 10.1109/MAP.2005.1487785. 42. Salimi, T, Maghoul, A, Abbasid, AA: Design of a compact Gaussian profiled corrugated horn antenna for low sidelobe-level applications. International Journal of Computer Theory and Engineering, Optics Express Vol. 20, Issue 9, 9371-9381 (2012). https://doi.org/10.1364/OE.20.009371

\section{List of abbreviations:}

'Not applicable'.

\section{Figure Captions:}

Fig. 1 Overview of the designed aspherical lens.

Fig. 2 Spot diagram of the designed aspherical lens.

Fig. 3 Example of a Fresnel lens: (a) Multi-dielectric Fresnel zone plate lens and (b) grooved Fresnel zone plate lens.

Fig. 4 Geometry used to determine the locations of transition points for a diffraction lens.

Fig. 5 Designed Fresnel lens.

Fig. 6 3D Design of Fresnel lens. 
Fig. 7 Optimized Fresnel lens spot diagram.

Fig. 8 Spillover loss of combined lens and Horn antenna.

Fig. 9 Schematic of the designed Gaussian profile horn with flat wall.

Fig. 10 Radiation pattern of flat wall Gaussian profile horn at frequency of $94 \mathrm{GHz}$.

Fig. 11 Schematic of the designed horn.

Fig. 12 Mode converter design.

Fig. 13 Phasing section design.

Fig. 14 Corrugated Gaussian section design.

Fig. 15 Radiation pattern of the designed corrugated Gaussian horn at frequency of 94 $\mathrm{GHz}$.

Fig. 16 Radiation pattern of the far field at a frequency of $94 \mathrm{GHz}$. 